\title{
Novel Dual-Band Single Circular Polarization Antenna Feeding Network for Satellite Communications
}

\author{
Carlos A. Leal-Sevillano, Jorge A. Ruiz-Cruz, José R. Montejo-Garai , Jesús M. Rebollar
}

\begin{abstract}
In this paper a novel dual-band single circular polarization antenna feeding network for satellite communications is presented. The novel antenna feed chain $^{1}$ is composed of two elements or subsystems, namely a diplexer and a bi-phase polarizer. In comparison with the classic topology based on an orthomode transducer and a dual-band polarizer, the proposed feed chain presents several advantages, such as compactness, modular design of the different components, broadband operation and versatility in the subsystems interconnection. The design procedure of this new antenna feed configuration is explained. Different examples of antenna feeding networks at $20 / 30 \mathrm{GHz}$ are presented. It is pointed out the excellent results obtained in terms of isolation and axial ratio.
\end{abstract}

Index Terms-Circular polarization, dual-band, waveguide, satellite communications, orthomode transducer, antenna, polarizer, diplexer, multibeam, K/Ka band.

\section{INTRODUCTION}

The increasing demand of satellite communications performance in terms of channel capacity has pushed the development of multibeam antennas [2]. Multiple spot beam systems are expected to be the most common scenario in new generation satellite communications [3]. These multibeam antennas are composed of a focal plane array illuminating a reflector antenna. The focal plane array is made up of a cluster of high efficiency horns and every single horn of the cluster has its own feed chain. Moreover, not all the horn feed chains are necessarily equal and different configurations are possible.

In regard to the electrical characteristics of the feed chains, satellite equipments maintain the dual-band specifications in $\mathrm{K} / \mathrm{Ka}$ bands for future communication systems; following the natural tendency of increasing the operation frequency with the subsequent mass and volume savings. Circular polarization is still required due to its advantages in terms of minimizing path loss and ease of antenna alignment.

In this new multibeam scenario different antenna feeding networks are desired:

- Gateway feed: dual-band double circular polarization, four ports feed.

- Tracking feed: tracking signals using the $T E_{11}, T M_{01}$ and $T E_{21}$ modes, sum and difference diagrams.

${ }^{1}$ Patent pending [1].



Figure 1. (a) Multibeam scenario. (b) Dual-band single circular polarization feed chain block diagram.

- User feed: dual-band single circular polarization, two ports feed.

The aforementioned feeds have also their homologous in the ground stations. However, the required electrical specifications might be different, e.g. $0.3 \mathrm{~dB}$ axial ratio and $50 \mathrm{~dB}$ inter-band isolation for on-board equipments and $0.7 \mathrm{~dB}$ axial ratio and $100 \mathrm{~dB}$ inter-band isolation for ground equipments.

The development of gateway feed chain has been carried out in the last years [3], [4], [5], [6]. The preferred alternative is based on using a six port self-diplexed waveguide junction combined with phase shifters, couplers and septum-polarizers. The complete antenna feed chain provides dual-band double circular polarization. The key element of this configuration is the self-diplexed junction. This element can be designed according to different high performance configurations such as, coaxial based orthomode junctions [7], asymmetric junction with dummy loads [8] and multifrequency enhanced turnstile junctions [9], [10].

Tracking feed chains are very important in the multibeam context. At least one tracking system is required in order to align and adjust the multibeam antenna coverage at the 
correct position. It should also be mentioned that combinations of the different feeds are also possible, such as include the tracking signals in the gateway feeds getting a combined gateway/tracking feed [3], [6].

The last required feed chain configuration is the user feed, or two ports feed, for dual-band single circular polarization. This configuration is intended to support the downlink and uplink frequency bands (typically $\mathrm{K}$ - and Ka-band for new generation satellite communications) with an orthogonal circular polarization for each band. This is expected to be the most used configuration since most of the beams demand this electrical characteristics, as well as, multiple satellite signal receivers at ground stations.

The classic implementation of the user feed chain is based on a dual-band orthomode transducer (OMT) [11] and a dualband polarizer [12], [13], both elements conveniently linked [14]. Nevertheless, this alternative presents serious drawbacks in terms of electrical design and practical implementation, such as narrow band, large axial dimension and performance degradation in terms of isolation and axial ratio when assembled.

A different approach already used in multibeam antennas is to implement the user feed with a gateway feed and matched loads at two of the ports. This configuration overcomes the performance issues of the conventional OMT and polarizer configuration. However, it is a cumbersome and rather expensive solution.

We present a novel user feeding network architecture [1], with several advantages against the classic option and therefore a suitable alternative for new satellite communication equipments, either on-board or on ground stations.

\section{PROPOSED ANTENNA FEED CHAIN}

In order to improve the electrical performance provided by the classic feed chain option consisting in a dual-band OMT and polarizer, we propose a new antenna feeding network concept illustrated in Fig. 1. The feed chain is composed of the following elements:

- Diplexer: to divide/combine the two frequency bands.

- Monomode waveguide: to link diplexer and bi-phase polarizer. It might include arbitrary bends and routing or even a flexible waveguide since it propagates a single mode.

- Bi-phase polarizer: to generate circular polarization, right hand circular polarization (RHCP) and left hand circular polarization (LHCP) at the first and second band, respectively or vice versa.

The two main elements of the feed chain are the diplexer and the bi-phase polarizer. It should be emphasized that in this configuration a single mode waveguide links both elements, unlike in the classic configuration. This fact leads to a modular design and assembly of the antenna feed. In other words, there is not performance degradation when diplexer and bi-phase polarizer are joined. The output waveguide of the polarizer, generally an square or circular waveguide, connects directly the horn antenna.

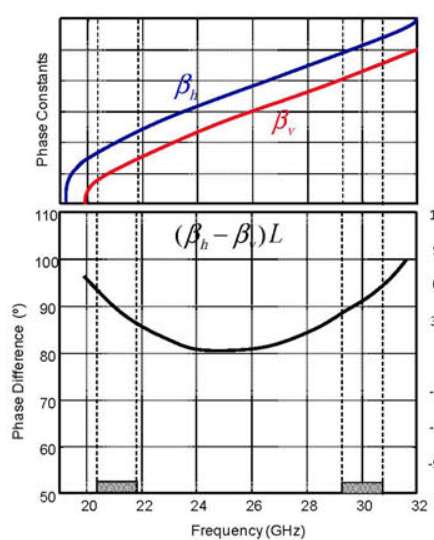

(a)

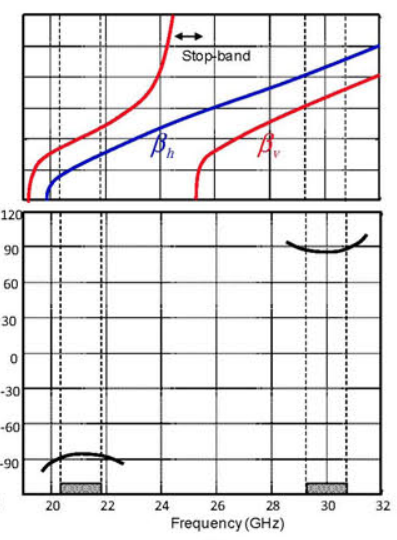

(b)
Figure 2. Schematic of propagation constants and phase difference for: (a) conventional $90^{\circ} / 90^{\circ}$ shifter and (b) $-90^{\circ} / 90^{\circ}$ bi-phase shifter.
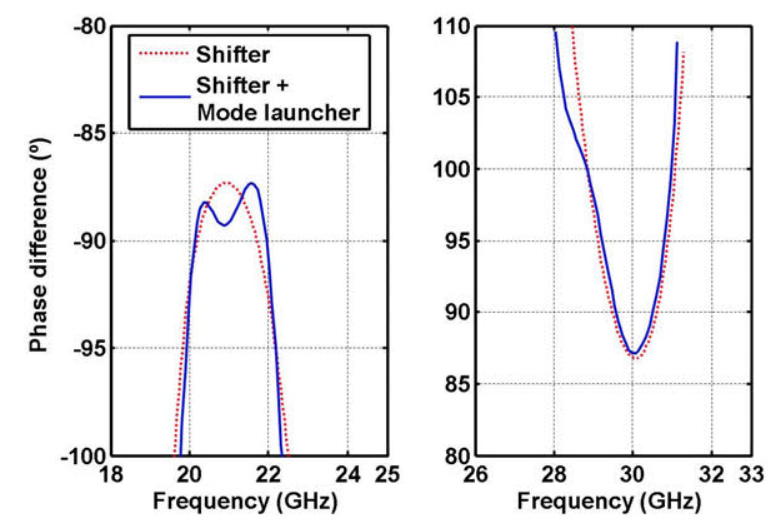

Figure 3. Phase difference between the orthogonal lineal polarizations at the output waveguide. Comparison between shifter (periodic waveguide) and shifter with mode launcher.

One of the two aforementioned main elements, the diplexer, is a well-known device and its design procedure, as well as, the fabrication process are well established. Diplexers are developed by different microwave components companies being commercially available. In this way the key element of the proposed antenna feed is the bi-phase polarizer.

\section{BI-PHASE POLARIZER DESIGN}

The bi-phase polarizer has two elements: a mode launcher that adjusts the linear polarization at the rectangular waveguide to the phase shifter and a bi-phase shifter based on a pseudoperiodic waveguide. An schematic of the bi-phase polarizer is shown in Fig. 1. The goal of this device is to transform the signal coming from the diplexer through a single mode waveguide, typically a rectangular waveguide propagating the $T E_{10}$, into a circularly polarized electromagnetic field at the output waveguide. Moreover, the circular polarization generated at the first and second band should be different, RHCP and LHCP, respectively, or vice versa. To this end, a phase shifter with $-90^{\circ} / 90^{\circ}$ phase difference between vertical and horizontal polarization at the first/second band is required. This bi-phase behavior has been previously obtained by means 
of periodic waveguides [15], [16]. It is important to distinguish between the bi-phase shifter required in the proposed feed, with $-90^{\circ} / 90^{\circ}$ phase difference, and the phase shifter normally used in the classic feeds based on corrugated waveguides [16] with $90^{\circ} / 90^{\circ}$ phase difference, Fig. 2.

\section{A. Periodic waveguide}

The initial predesign is critical in the final polarizer performance, in particular, in order to achieve the required $-90^{\circ} / 90^{\circ}$ phase difference. This predesign is based on a rigorous study of the propagation characteristics in periodic waveguides.

It is known that corrugated waveguides used for conventional $90^{\circ} / 90^{\circ}$ phase shifters have a characteristic curve as shown in Fig. 2 due to the propagation constants of the vertical and horizontal polarizations. The shape of this phase difference curve cannot be significantly modified. This is the reason of the narrow band performance in the classic feed networks.

In order to obtain the $-90^{\circ} / 90^{\circ}$, different periodic waveguides should be used, such as the geometries presented in Figs. 4 and 7, using waveguides with high height/width ratio in the cross section. This kind of periodic waveguides can be adjusted to introduce a stop band between both bands for one of the polarizations, as illustrated in Fig. 2. In that way, a bi-phase behavior in the phase difference is obtained. Notice that the magnitude of the phase curve for each band is similar to the shape of the $90^{\circ} / 90^{\circ}$ phase shifter in the whole bandwidth. This fact allows to significantly increase the operation bandwidth, as well as, the separation between bands. In Fig. 2 a comparison between the propagation constants and the phase differences for a conventional and for a bi-phase shifter is presented.

The initial dimensions and number of periods of the phase shifter are obtained from the periodic waveguide analysis.

\section{B. Mode launcher}

The second element of the polarizer is the mode launcher directly attached to the bi-phase shifter. This transition adequate the electromagnetic field at the input single mode waveguide to the input of the phase shifter. Although, this elements might seem just a mere waveguide transformer, it has a crucial impact in the axial ratio of the polarizer.

When the single mode input waveguide of the polarizer is excited, at the waveguide between mode launcher and phase shifter, Fig. 4, there exist an small cross coupling between orthogonal lineal polarization scattered back to the mode launcher. This signal is again scattered forward to the shifter since the input waveguide is cut-off for this polarization. All this produces certain change in the phase difference at the output of the polarizer, as can be observed in Fig. 3.

Therefore, the mode launcher introduces a small ripple in the phase difference of the polarizer, Fig. 3. This ripple can be used to improve the purity of the output circular polarization. In that way, the final dimensions of the polarizer (mode launcher and phase shifter) can be adjusted to obtain an equiripple in the phase difference around $-90^{\circ} / 90^{\circ}$. This is illustrated in Fig. 3 for the first example considered in the next section.

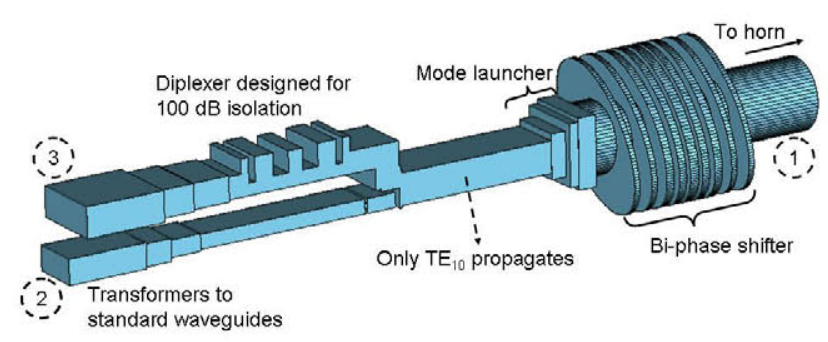

Figure 4. Geometry of the first antenna feed chain design.

Table I

FIRST ANTENNA FEED CHAIN RESULTS.

\begin{tabular}{|c|c|c|}
\hline & \\
\hline & Band 1 & Band 2 \\
\hline Frequency & $20-22 \mathrm{GHz}$ & $29.25-30.75 \mathrm{GHz}$ \\
\hline Polarization & RHCP or LHCP & LHCP or RHCP \\
\hline Return Loss & $>21 \mathrm{~dB}$ & $>23 \mathrm{~dB}$ \\
\hline AR & $<0.6 \mathrm{~dB}$ & $<0.6 \mathrm{~dB}$ \\
\hline XPD & $>30 \mathrm{~dB}$ & $>30 \mathrm{~dB}$ \\
\hline \multirow[t]{2}{*}{ Isolation } & \multicolumn{2}{|c|}{ Band $1-B$ and $2>100 \mathrm{~dB}$} \\
\hline & \multicolumn{2}{|c|}{ Band $2-$ Band $1>100 \mathrm{~dB}$} \\
\hline \multirow[t]{2}{*}{ Interfaces } & WR-42 & WR-28 \\
\hline & \multicolumn{2}{|c|}{ Common port: Circular Waveguide } \\
\hline
\end{tabular}

\section{ANTENNA FEED CHAIN EXAMPLES}

Different examples are presented to illustrate the potential of the proposed antenna feeding network. All the designs operate at $20 / 30 \mathrm{GHz}$ (around $40 \%$ fractional bandwidth separation) but with different bandwidth and electrical specifications.

\section{A. First example}

As first example of the proposed antenna feed, we have carried out a design with the specifications summarized in Table I. It should be mentioned the high isolation specified which is normally required for ground user feeds.

The diplexer is based on high-pass and low-pass filters designed for $100 \mathrm{~dB}$ inter-band rejection. The high-pass filter is a cut-off waveguide at the lower band and the low-pass filter is an asymmetric corrugated filter with five E-plane stubs. This is a very appropriate topology for the prescribed bands separation leading to the desired high isolation. The monomode waveguide between diplexer and polarizer is a rectangular waveguide, which only propagates the $T E_{10}$ mode.

For the bi-phase polarizer design the above described design procedure has been followed. In this design elliptical and circular waveguides have been selected. A total of ten elliptical waveguides in the phase shifter were required to achieve the desired $0.6 \mathrm{~dB}$ axial ratio in both bands.

The full-wave design and optimization of the individual components, as well as the complete antenna feed simulation has been carried out by means of an efficient in-house made mode-matching code. The waveguides involved in the feed chain are rectangular, circular and elliptical. The modal spectrum of all these waveguides is computed analytically using trigonometric, Bessel and Mathieu functions. Then the generalized scattering matrix (GSM) of every step are calculated and cascaded leading to the GSM of the complete feed. 


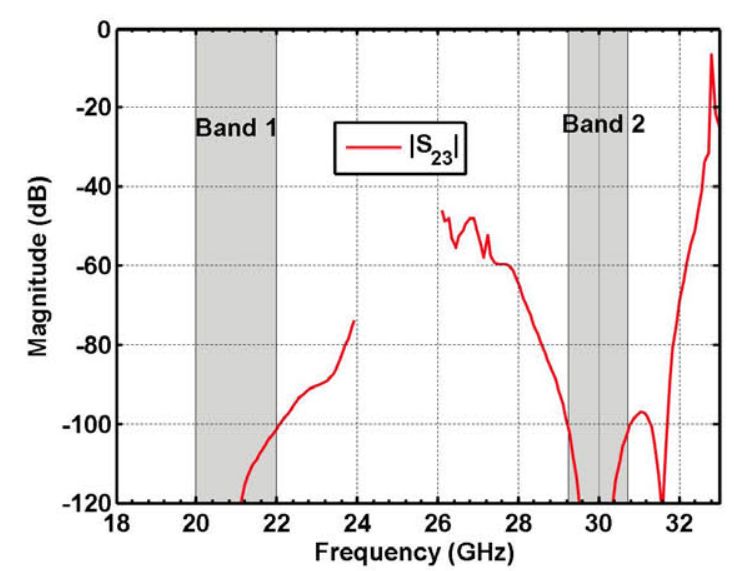

Figure 5. Simulated isolation of the first antenna feed design.

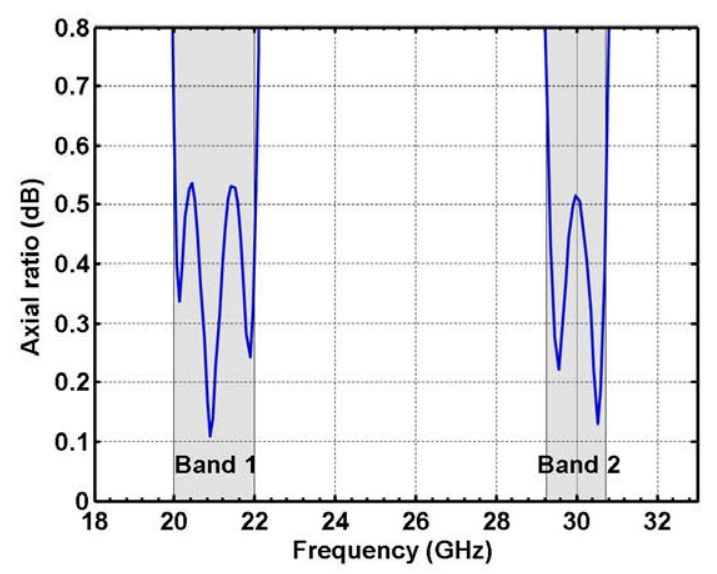

Figure 6. Simulated axial ratio of the first antenna feed design.

The geometry of the final antenna feeding network is shown in Fig. 4. The simulated isolation and axial ratio of the complete feed chain are shown in Figs. 5 and 6, respectively. The results show the expected values summed up in Table I. An important result to be noticed are the excellent values of $100 \mathrm{~dB}$ isolation and $0.6 \mathrm{~dB}$ axial ratio obtained. Moreover, isolation and axial ratio are uniquely determined by the diplexer and the polarizer, respectively. When both elements are joined no performance degradation is observed due to the single mode waveguide. This result is very important and allows a modular design of the feed chain. On the contrary, the above modular design is not feasible for the conventional OMT plus polarizer feed scheme.

\section{B. Second example}

In the second example a feed chain design with the characteristics summarized in Table II has been carried out following the aforementioned design procedure. In this case the selected bands and bandwidth are a very common specification used in satellite communications.

The diplexer is based on a high-pass and a corrugated symmetric low-pass filter with the main driving specification of $100 \mathrm{~dB}$ isolation between frequency bands. In this case the
Table II

SECOND ANTENNA FEED CHAIN RESULTS.

\begin{tabular}{|c|c|c|}
\cline { 2 - 3 } \multicolumn{1}{c|}{} & Band 1 & Band 2 \\
\hline Frequency & $18.2-20.2 \mathrm{GHz}$ & $28-30 \mathrm{GHz}$ \\
\hline Polarization & RHCP or LHCP & LHCP or RHCP \\
\hline Return Loss & $>21 \mathrm{~dB}$ & $>23 \mathrm{~dB}$ \\
\hline AR & $<0.6 \mathrm{~dB}$ & $<0.6 \mathrm{~dB}$ \\
\hline XPD & $>30 \mathrm{~dB}$ & $>30 \mathrm{~dB}$ \\
\hline Isolation & \multicolumn{2}{|c|}{ Band 1 - Band 2 $>100 \mathrm{~dB}$} \\
\cline { 2 - 3 } & \multicolumn{2}{|c|}{ Band 2 - Band 1 $>100 \mathrm{~dB}$} \\
\hline Interfaces & Common port: Circular Waveguide \\
\hline
\end{tabular}



Figure 7. Simulated axial ratio of the second antenna feed design.

bi-phase shifter is based on a pseudo periodic waveguide made up of alternated circular and rectangular waveguides, with a total of twelve rectangular waveguide sections. The mode launcher is a rectangular to circular waveguide transition. The complete polarizer is optimized in order to fully take advantage of the mode launcher and phase shifter interaction. In this case the bi-phase polarizer is intended to extend the bands separation and increase the bandwidth to cover the whole assigned satellite uplink and downlink bands.

The geometry of the final antenna feed design is shown in the inset of Fig. 7. The achieved electrical specifications are summarized in Table II. The axial ratio is shown in Fig. 7. It is important to highlight the wide bandwidth presented in this case with $2 \mathrm{GHz}$ at the lower and upper frequency bands for an axial ratio below $0.6 \mathrm{~dB}$.

Another important results to be noticed is the unusual shape of the axial ratio curves presented in Fig. 7. This is mainly due to the ripple in the phase difference of the bi-phase polarizer (mode launcher plus phase shifter) as discussed before, see Fig. 3.

\section{Third example}

For some on-board equipments narrow band but stringent axial ratio and return loss specifications are required. The last example is a geometry similar to the previous one but in this case the bi-phase polarizer is designed for an axial ratio bellow $0.2 \mathrm{~dB}$ in a narrower band. The final performance is detailed in Table III. Fig. 8 shows the simulated axial ratio with a 
Table III

THIRD ANTENNA FEED CHAIN RESULTS.

\begin{tabular}{|c|c|c|}
\cline { 2 - 3 } \multicolumn{1}{c|}{} & Band 1 & Band 2 \\
\hline Frequency & $19.6-20.3 \mathrm{GHz}$ & $29.4-30.1 \mathrm{GHz}$ \\
\hline Polarization & RHCP or LHCP & LHCP or RHCP \\
\hline Return Loss & $>30 \mathrm{~dB}$ & $>30 \mathrm{~dB}$ \\
\hline AR & $<0.17 \mathrm{~dB}$ & $<0.17 \mathrm{~dB}$ \\
\hline XPD & $>40 \mathrm{~dB}$ & $>40 \mathrm{~dB}$ \\
\hline Isolation & Band 1 - Band 2 $>100 \mathrm{~dB}$ \\
\cline { 2 - 3 } & \multicolumn{2}{|c|}{ Band 2 - Band 1 > 100 dB } \\
\hline Interfaces & Common port: Circular Waveguide \\
\hline
\end{tabular}

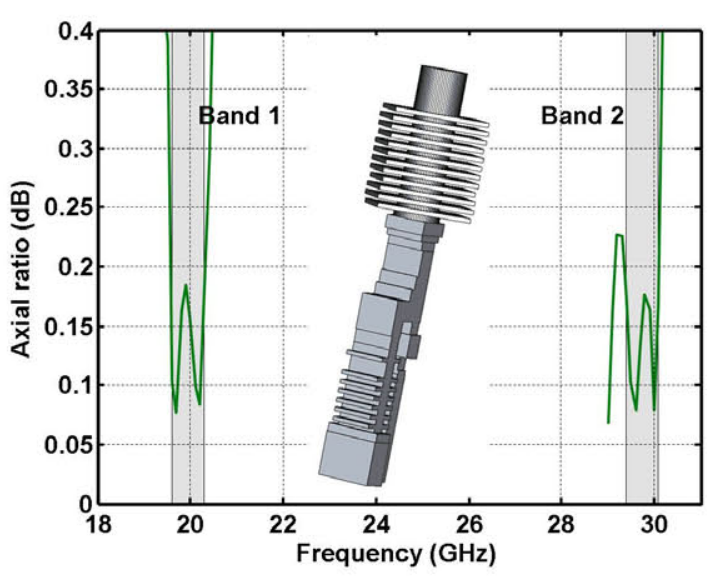

Figure 8. Simulated axial ratio of the third antenna feed design.

maximum level of $0.17 \mathrm{~dB}$ in the two $700 \mathrm{MHz}$ operating bands.

\section{DISCUSSION AND CONCLUSIONS}

A new antenna feeding network for dual-band single circular polarization has been presented. The main motivation of this architecture is to overcome the important issues of the conventional alternative and at the same time to avoid the use of much cumbersome and expensive options that are not intended, in principle, for single polarization applications.

As a new feed alternative it should be compared with the classic solution of a dual-band OMT an polarizer.

The conventional feed has an intrinsic limitation in terms of bandwidth and separation between bands. This is caused by the phase difference of the corrugated waveguide polarizers. Band separation and bandwidth are improved with the bi-phase polarizer.

There is a minimum length between OMT and polarizer to attenuate higher order modes. Moreover, OMT and polarizer must be aligned. The proposed feed uses a single mode waveguide to join diplexer and bi-phase polarizer. In that way, the axial length can be reduced. In addition, diplexer and polarizer are not necessary aligned in the axial direction.

The electrical performance, particularly isolation and axial ratio, deteriorates when OMT and polarized are assembled. The presented feed allows a modular design and the electrical characteristics are well established by each particular component.
In summary, the main advantages of the new configuration are to overcome the bandwidth limitations imposed by the classic topology, compactness and versatility in the components interconnection and modular design and assembly of the different feed chain elements.

We believe that the proposed antenna feed has a huge potential for satellite communication equipments and in particular in the context of new generation multibeam antennas.

\section{ACKNOWLEDGMENT}

This work has been supported by the Spanish government program TEC2010-17795, the CONSOLIDER CSD200800068 and the Universidad Politécnica de Madrid under a Ph.D. grant.

\section{REFERENCES}

[1] C. A. Leal-Sevillano, J. M. Rebollar, J. A. Ruiz-Cruz, and J. R. MontejoGarai, Spanish Patent Application, P 6096/2013, Sep. 24, 2013.

[2] K. S. Rao and M. Q. Tang, "Stepped-reflector antenna for dual-band multiple beam satellite communications payloads," IEEE Transactions on Antennas and Propagation, vol. 54, pp. 801-811, 2006.

[3] E. Reiche, S. Stirland, C. Hartwanger, U. P. Hong, R. Gehring, and H. Wolf, "A dual circular combined K/Ka-band RF sensing feed chain for multi beam satellite antennas," in Proceedings of the 5th European Conference on Antennas and Propagation (EUCAP), 2011.

[4] P. Sarasa, A. Baussois, and P. Regnier, "A compact single-horn C/X dual band and circular polarized Tx Rx antenna system," in IEEE Antennas and Propagation Society International Symposium, 2004.

[5] R. A. Hoferer and R. Schwerdtfeger, "Development of a wide-band quadrature junction for simultaneous C-band and Ku-band satellite communication applications," in Antennas and Propagation Society International Symposium, 2002. IEEE, vol. 4, 2002, pp. 116-119 vol.4.

[6] E. Amyotte, Y. Demers, L. Hildebrand, M. Forest, S. Riendeau, S. SierraGarcia, and J. Uher, "Recent developments in Ka-band satellite antennas for broadband communications," in Proceedings of the Fourth European Conference on Antennas and Propagation (EuCAP), 2010.

[7] I. M. Davis, C. Granet, J. S. Kot, G. Pope, and T. Mellor, "A simplified simultaneous X/Ka-band feed-system design," in IEEE Military Communications Conference, MILCOM, 2008, pp. 1-5.

[8] C. Lee-Yow, "High performance antenna feeds and components for space application," in First European Conference on Antennas and Propagation (EuCAP), 2006.

[9] J. R. Montejo-Garai, J. A. Ruiz-Cruz, C. A. Leal-Sevillano, and J. M. Rebollar, "Modelling of dual-polarisation diplexers based on enhanced multiport turnstile junctions," IET Microwaves, Antennas Propagation, vol. 7, no. 7, pp. 485-492, 2013.

[10] D. Garcia, V. Cabrera, and J. M. M. Ruiz, "High performance of dual band/dual polarization compact OMT," in IEEE-APS Topical Conference on Antennas and Propagation in Wireless Communications (APWC), 2013, pp. 703-706.

[11] J. A. Ruiz-Cruz, J. R. Montejo-Garai, and J. M. Rebollar, "Optimal configurations for integrated antenna feeders with linear dual-polarisation and multiple frequency bands," IET Microwaves, Antennas Propagation, vol. 5, no. 8, pp. 1016-1022, Jun. 2011.

[12] J. M. Rebollar and J. de Frutos, "Dual-band compact square waveguide corrugated polarizer," in IEEE Antennas and Propagation Society International Symposium, vol. 2, Aug. 1999, pp. 962-965.

[13] C. A. Leal-Sevillano, J. R. Montejo-Garai, J. M. Rebollar, and J. A. Ruiz-Cruz, "CAD for dual-band polarizers in corrugated rectangular waveguide," in 41st European Microwave Conference (EuMC), 2011.

[14] U. Rosenberg and M. Schneider, "Power splitting transition for circularly polarized feed networks," IEEE Microwave and Guided Wave Letters, vol. 10, no. 8, pp. 307-309, 2000.

[15] J. M. Rebollar and J. Esteban, "CAD of corrugated circular-rectangular waveguide polarizers," in Eighth International Conference on Antennas and Propagation, 1993.

[16] C. A. Leal-Sevillano, J. A. Ruiz-Cruz, J. R. Montejo-Garai, and J. M. Rebollar, "Dual-band bi-phase waveguide polarizer for a novel feeder network without orthomode transducer," in 43st European Microwave Conference (EuMC), 2013. 